\title{
CT STUDY COMPARING SULCAL CHANGES AND THIRD VENTRICLE SIZE AS AGE PROGRESSES FROM 40 TO 80
}

\author{
Vinu C. George ${ }^{1}$, Jose Manuel2 ${ }^{2}$ Biju Mohan³, Indi Kusian4, Sourabh A. P5, Arun V. J6
}

\section{HOW TO CITE THIS ARTICLE:}

Vinu C. George, Jose Manuel, Biju Mohan, Indi Kusian, Sourabh A.P, Arun V. J. “CT Study comparing Sulcal Changes and Third Ventricle size as age progresses from 40 to 80". Journal of Evolution of Medical and Dental Sciences 2014; Vol. 3, Issue 44, September 15; Page: 10778-10785, DOI: 10.14260/jemds/2014/3404

\begin{abstract}
INTRODUCTION: Computerized tomography, also called CT combines a series of X-ray views taken from many different angles and computer processing to create cross-sectional images of the bones and soft tissues inside your body. It has brought about tremendous changes in the field of diagnostic and research medicine. Here CT is used to measure the various dimensions of 6 selected sulci of brain and third ventricle. With the recent advances in technology, sulcal pattern and development is being studied extensively to understand the functioning of brain. There is evidence of sulcal dimensional changes as age of an individual progress. This may be responsible for behavioral or intellectual changes in a individual. Through this study I intend to understand how the sulcal dimension may vary with the help of CT scans. 6 sulci were selected and their width was measured in 80 individuals between the age group of 40 and 80 . Through statistical analysis the data will reveal any recognizable changes in the width of the selected sulcus with progression of age. The third ventricle size may also be influenced by the age of the individual. Using the help of CT scan I am intending to measure the dimensions of the third ventricle. Recent studies have shown that size and volume of the third ventricle have significant role in the geriatric population. AIMS AND OBJECTIVES: To study the age related changes in the width of left and right central sulcus, superior temporal sulcus, parieto-occipital sulcus and to study the age related changes in third ventricle depth, length and width through the use of CT scans. STUDY SETTINGS: A cross-sectional study comprising of 60 patients coming to the radiology department for the C T scan. The CT scans for the study were taken randomly between the ages of 40 to 80. RESULTS: The purpose of the study was to study the changes in the dimensions of sulci and the third ventricle of the brain. Hypothesis was that the sulcal width increases with advance in age. the objective was to analyze the data from CT scans and bring out the variation in sulcal depth and third ventricle. Positive correlations were noted in the width of right central sulcus, left and right superior frontal sulcus, right and left parieto-occipital sulcus and width of the third ventricle. No relationship was noted between age and the width of left central sulcus, depth and length of third ventricle.
\end{abstract}

KEYWORDS: Third ventricle, Brain Sulcus Computerized tomography,

INTRODUCTION: Computerized tomography, also called CT combines a series of X-ray views taken from many different angles and computer processing to create cross-sectional images of the bones and soft tissues inside your body. It has brought about tremendous changes in the field of diagnostic and research medicine.

Here CT is used to measure the various dimensions of 6 selected sulci of brain and third ventricle. Sulcus is a groove or depression in the cerebral cortex. It surrounds a gyrus, creating the characteristic folded appearance of the brain in humans and other mammals. Even though these sulci and gyri follow a specific pattern it varies from individual to individual. 
Human brain structure vary greatly from the animal brains and that is makes human unique and be aware of himself and his surroundings. With the recent advances in technology, sulcal pattern and development is being studied extensively to understand the functioning of brain. There is evidence of sulcal dimensional changes as age of an individual progress.

This may be responsible for behavioral or intellectual changes in an individual. Through this study I intend to understand how the sulcal dimension may vary with the help of CT scans. 6 sulci was selected and their width was measured in 80 individuals between the age group of 40 and 80 . Through statistical analysis the data will reveal any recognizable changes in the width of the selected sulcus with progression of age. [Fig. 1]

The third ventricle is one of four ventricles in the brain that communicate with one another. The third ventricle sends messages to and receives messages from the lateral ventricles, which is located in front of the third ventricle, and the aqueduct of the midbrain, which is located directly behind the third ventricle.

It is filled with cerebrospinal fluid and helps to protect the brain from injury. It is a narrow cavity that is located between the two lateral halves of the brain. The third ventricle is involved in several functions of the body including: Protection of the brain from trauma [Fig. 1]

The third ventricle size may also be influenced by the age of the individual. Using the help of CT scan I am intending to measure the dimensions of the third ventricle. Recent studies have shown that size and volume of the third ventricle have significant role in the geriatric population.

All the study subjects were patients with normal CT scans without any pathology, so that there will be minimal variation to the outcome. The study will help in understanding the neurological changes and their effect on the humans.

REVIEW OF LITERATURE: Maryam E. Rettmann et al(1) from the National Institute on Aging, National Institutes of Health, Baltimore, USA found in their study that more shallow sulci could signify that older individuals have more open sulci than younger individuals, which is an age associated cortical shape differences.

They concluded that the most consistent findings are decreased in central mean thickness, decrease in central GM volume, decrease in cingulate GM volume, and decrease in cingulate high positive curvature points. The changes in cortical thickness may be associated with the sulcal width changes in old age people.

On an another study by Peter Kochunov(3) at Research Imaging Center, University of Texas Health Science Center at San Antonio, San Antonio, Texas found highly significant increase in sulcal width and decrease in sulcal depth with age were observed.

The overall rate of increase in sulcal width with age was $0.71 \mathrm{~mm} /$ decade, which was about twice the rate of age-related decrease in sulcal depth, $0.38 \mathrm{~mm} /$ decade. Study by Peter Kochunov and et.al points that there seems to be significant sulcal width and depth changes in study at texas.

The same is intended to be done here on a different scale. Vincent A. Magnotta(4) et al through their research suggests that the aging process affects gyrification, with the brain appearing more 'atrophic' with increasing age.

The shape of gyri and sulci change significantly over time, with the gyri becoming more sharply and steeply curved, while the sulci become more flattened and less curved. Cortical thickness also decreases over time. Cortical thinning progresses more rapidly in males than in females. 
The progression of these changes appears to be relatively stable during midlife and to begin to progress some time during the fourth decade. Tang $\mathrm{Y}^{(5)}$ et al investigated the longitudinal agerelated changes in human brain volume using stereological methods. The average volume of the lateral ventricles on the second MRI was increased by $5.6 \%+/-3.6 \%$ per year.

A study by Dr. Liju S Mathew, ${ }^{(2)}$ measured the parameters of third ventricle using MRI of 150 male and females in north India. They fund that width anterior to the interthalamic adhesion has no statistical significance and posterior is significant among 6-9 years.

They concluded that measurement from birth to 30 years of age, the third ventricle has clinical implication. In my study with CT scans, I will measure the depth, length and width of third ventricle and assess its changes in a people of age group $40 \mathrm{t} 80$.

\section{AIMS AND OBJECTIVES:}

- To study the age related changes in the width of left and right central sulcus, superior temporal sulcus, parietooccipital sulcus.

- To study the age related changes in third ventricle depth, length and width through the use of CT scans.

\section{MATERIALS AND METHODOLOGY:}

STUDY SUBJECTS: The proposed work will be a cross-sectional study comprising of 60 patients coming to the radiology department for the $\mathrm{C}$ T scan. The CT scans for the study were taken randomly between the ages of 40 to 80 . No personal data of the patient was misused.

EXCLUSION CRITERIA: Individuals with former lesions, head trauma, endocrinopathies, chromosomal abnormalities, non-heterosexuals, cerebrovascular accidents, and other cerebral problems. All details were sorted out and the study was approved by our institutional review board. Ethics committee has approved the research methodology.

The study subjects were grouped into two groups. Group 1 comprising of age between 40 to 60 and Group 2 of age between 60 to 80 .

MEASUREMENT OF DEPTH OF SULCI AND THIRD VENTRICLE: For the purpose of measuring the depth 5 main sulcal region were selected. Those sulcal regions include-the buried cortex surrounding the left and right central, superior frontal, and parietooccipital sulci. The width is defined as the length of the shortest path, between the edges of the above mentioned sulcus.

Points for antero-posterior length and height were best appreciated on the cross sectional slice, the anterior point marked on the lamina terminalis, the posterior point on the posterior commissure points used in reference for antero-posterior length. The superior point is the highest curvature of the inferior surface of fornix and inferior point, marked on the superior surface of the mamillary body to measure the superior-inferior height.

Point on the right lateral wall of the third ventricle and an identical point on the left lateral wall on thalamus points the width anterior. A point on the right lateral wall of the third ventricle and another identical point on the left lateral wall on thalamus is used to measure width posterior to interthalamic adhesion.(2) 
STATISTICAL ANALYSIS: Mean values of width of left and right central sulcus, superior frontal sulcus, parietooccipital sulcus. Mean values of length, width and depth is calculated from the collected data. They are then categorized as two, age group between 40 to 60 and age group between 60 to 80 .

SSPE software was used to find the significance in width of each sulcus and dimensions of third ventricle using student $t$ test. One variable from both the groups were compared. For the purpose of statistical analysis of 2 variables of 2 groups, we use the software of ANOVA.(6)

Analysis of variance (ANOVA) is a collection of statistical models used to analyze the differences between group means and their associated procedures (such as "variation" among and between groups. In the ANOVA setting, the observed variance in a particular variable is partitioned into components attributable to different sources of variation.

\section{RESULTS:}

\section{STUDENT T TEST:}

\section{Left central Sulcus:}

[Table.1] Null hypothesis: there exists no significant relationship between the width of left central sulcus of group 1 and 2. Null hypothesis was accepted. From the student t test, we were able to see that the $\mathrm{p}$ value is less than 0.005 and hence not significant. Hence, there exist no specific relationship between the age and the width of the left central sulcus.

\section{Right central sulcus:}

[Table.2] Null hypothesis: there exists no significant relationship between the width of right central sulcus of group 1 and 2. Null hypothesis was rejected. The student t test reveals the significance between group 1 and group 2 right central sulcus. The p value of 0.005 indicates the significant relationship. It can be observed that the relationship between the age and the right central sulcus is significant.

3. Left superior frontal sulcus:

[Table.3] Null hypothesis: there exists no significant relationship between the width of left superior frontal sulcus of group 1 and 2 . Null hypothesis rejected. P value of 0.001 indicates that left superior frontal sulcus between the age group of 40-60 and 60-80 have significant relationship. The results show that the relation between age and width of the left superior frontal sulcus is valid.

\section{Right superior frontal sulcus:}

[Table.4] Null hypothesis: there exists no significant relationship between the width of right superior sulcus of group 1 and 2. Null hypothesis was rejected. From the data shown it can be identified that there is significant relation between the right superior frontal sulcus of group 1 and 2 . Hence it can be seen that the right superior frontal sulcus is influenced by the age progression.

5. Left parietooccipital sulcus:

[Table.5] Null hypothesis: there exists no significant relationship between the width of left parietooccipital sulcus of group 1 and 2. Null hypothesis rejected. From the student $t$ test, it was seen that the $p$ value is less than 0.005 and hence there exist significant relationship between parietooccipital sulcus of group 1 and 2 . The relationship between the age and left parietooccipital sulcus width is strong. 


\section{ORIGINAL ARTICLE}

\section{Right parietooccipital sulcus:}

[Table.6] Null hypothesis: there exists no significant relationship between the width of right parietooccipital sulcus of group 1 and 2. Null hypothesis accepted. P value greater than 0.005 indicates that there is no significant relationship between the width of right parietooccipital sulcus of group 1 and 2 . There exist no specific relationship between the age and the width of the right parietooccipital sulcus.

7. Third ventricle depth:

[Table.7] Null hypothesis: there exists no significant relationship between the depth of third ventricle of group 1 and 2. Null hypothesis accepted. P value greater than 0.005 indicates that there is no significant relationship between the depth of third ventricle between the group of 1 and 2 . The depth of the third ventricle is not influenced by the changes in the age.

\section{Third ventricle length:}

[Table.8]Null hypothesis: there exists no significant relationship between the length of third ventricle of group 1 and 2. Null hypothesis accepted. $P$ value greater than 0.005 indicates that there is no significant relationship between the length of the third ventricle of group 1 and 2 . The length of the third ventricle and the age has no relationship.

\section{Third ventricle width:}

[Table.9] Null hypothesis: there exists no significant relationship between the width of third ventricle of group 1 and 2 . Null hypothesis rejected. P value less than 0.005 indicates that there is significant relationship between the width of the third ventricle between the group 1 and 2 . There exist a very high significant relationship between the age of the person and the width of the third ventricle.

DISCUSSION: Positive correlations were noted in the width of right central sulcus, left and right superior frontal sulcus, right and left parietooccipital sulcus and width of the third ventricle. No relationship was noted between age and the width of left central sulcus, depth and length of third ventricle.

The study shows significant age related changes in the width of right central sulcus in the age group of 40-60 and 60-80. This could be due to the aging process is associated with several structural, chemical, and functional changes in the brain as well as a host of neurocognitive changes. Maryam E Rettmann on her Cross-sectional and Longitudinal Analyses of Anatomical Sulcal Changes Associated with Aging noted Decreases in mean thickness of the central sulcus.

Left superior frontal sulcus shows strong correlation with the age of the person. The sulcal width seems to be increasing with age. Recent report in model organisms suggests that as organisms age, there are distinct changes in the expression of genes at single neuron level. Studies by Peter Kochunov had concluded that the sulcal widening could be associated with the increase in hyperintense white matter lesions.

He also noted the sulcal span earlier increase at the rate of $0.5 \mathrm{~mm} / \mathrm{cm}^{3}$ and later at the rate of $0.1 \mathrm{~mm} / \mathrm{cm}^{3}$. This could be due to the distinct changes in the expression of genes at single neuron level leading to widening of sulcal span. A positive correlation was recorded with the right superior frontal sulcus and age. The sulcal width increased with the progress in age. Research by Maryam $\mathrm{E}$ Rettmann shows negative correlation between the age and sulcal span. This could be due to the increased prevalance of right handed people and their left hemisphere is dominant. 
Various biochemical changes, circulatory and physiological changes in the geriatric population will result in the changes in the width of the right superior frontal sulcus. A positive correlation was noted between the right and left parietooccipital sulcus and the age of the individual. Both the sulcal width increased with the progress in age.

Maryam E Rettmann noted a negative correlation in the width of the left parietooccipital sulcus in contrast to the study here. He postulates this could be due to the less sharply folded sulcus in the older individual which is not so evident in the study subjects here. Third ventricle width showed very high significant relationship with the age of the individual in the study conducted.

The length and depth of the third ventricle were found to be non-significant relationship with age. Study by Liju S Mathew in the Christian medical college of Ludhiana gives the width of anterior to interthalamic adhesion to $5.38 \pm 1.33 \mathrm{~mm}$ in adult male and $5.73 \pm 1.61 \mathrm{~mm}$ in adult females. This could be due to the circulatory insufficiency and changes in the grey matter volume of the brain in old age.

CONCLUSION: The purpose of the study was to study the changes in the dimensions of sulci and the third ventricle of the brain. Hypothesis was that the sulcal width increases with advance in age. The objective was to analyze the data from CT scans and bring out the variation in sulcal depth and third ventricle. CT scans of the patients coming to the radiology department of the hospital were obtained following which left and right central sulcus, superior frontal sulcus, parietooccipital sulcus width and depth length and width of third ventricle was assessed and recorded. Anova software was used to analyse the variance in data.

From the study right central sulcus width for age 40 to 60 is $2.67+\backslash-.11$ and for 60 to 80 is $3.10+\backslash-.10$. Left superior frontal sulcus recorded $2.80+\backslash-0.12$ for age 40 to 60 and $3.50+\backslash-0.15$ for age 60 to 80. Right superior frontal sulcus measured 2.93+\-.11 for age 40 to 60 and $3.47+\backslash-0.17$ for age 60 to $80.2 .40+\backslash-0.09$ for age 40 to 60 and $2.70+\backslash-0.10$ for age 60 to 80 was measured for left parietooccipital sulcus. For the age group of 40 to 60 , the right parietooccipital sulcus measured $2.53+\backslash-0.10$ and for the age group 60 to $802.77+\backslash-0.09$ was recorded. The third ventricle width was measured as $3.10+\backslash-0.17$ for age 40 to 60 and $4.67+\backslash-0.20$ for the age group of 60 to 80 .

\section{REFERENCES:}

1. Maryam E. Rettmann, Michael A. Kraut, Jerry L. Prince, Susan M. Resnick. Cross-sectional and Longitudinal Analyses of Anatomical Sulcal Changes Associated with Aging. November 2006. 16(11).p" 1584-94.

2. Dr. Liju S Mathew, Prof. Molly Paul, Dr. Sandeep Bakshi. Assessment of Third Ventricle with MRI: Morphological Variations among Different Gender and Age Group. May 2012. 3(5).

3. Peter Kochunov, Jean-François Mangin, Thomas Coyle, Jack Lancaster, Paul Thompson, Dennis Rivière, et al. Age-related morphology trends of cortical sulci. Human Brain Mapping. November 2005. 26(3), pages 210-20.

4. Vincent A. Magnotta, Nancy C. Andreasen, Susan K. Schultz, Greg Harris, Ted Cizadlo, Dan Heckel, Peg Nopoulos, et al. Quantitative In Vivo Measurement of Gyrification in the Human Brain: Changes Associated with Aging. March 1999; 9(2):151-60.

5. Tang Y, Whitman GT, Lopez I, Baloh RW. Brain volume changes on longitudinal magnetic resonance imaging in normal older people. October 2001:11 (4);393-400. 


\section{ORIGINAL ARTICLE}

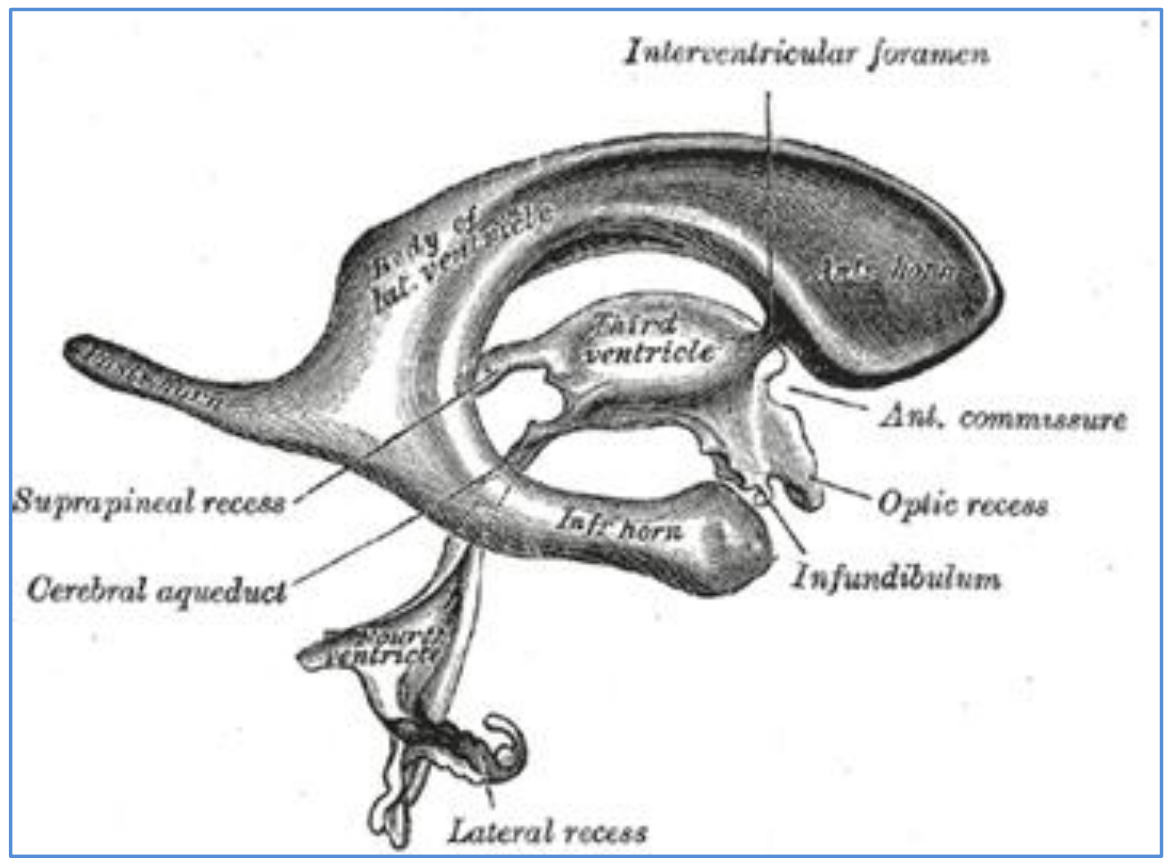

Fig.1: Pathway for the circulation of cerebrospinal fluid

\begin{tabular}{|c|c|c|c|}
\hline $\mathbf{T}$ & Degree of freedom & P value & Implication \\
\hline 1.621 & 58 & 0.11. & Not significant \\
\hline \multicolumn{3}{|c|}{ Table 1: Left central sulcus } \\
\hline
\end{tabular}

\begin{tabular}{|c|c|c|c|}
\hline $\mathbf{T}$ & Degree of freedom & P value & Implication \\
\hline 2.904 & 57 & 0.005 & Significant \\
\hline \multicolumn{4}{|c|}{ Table 2: Right central sulcus } \\
\hline
\end{tabular}

\begin{tabular}{|c|c|c|c|}
\hline $\mathbf{T}$ & Degree of freedom & P value & Implication \\
\hline 3.633 & 58 & 0.001 & Significant \\
\hline \multicolumn{4}{|c}{ Table 3: Left superior frontal sulcus }
\end{tabular}

\begin{tabular}{|c|c|c|c|}
\hline T & Degree of freedom & P value & Implication \\
\hline 2.575 & 51 & 0.013 & Significant \\
\hline \multicolumn{4}{|c|}{ Table 4: Right superior frontal sulcus } \\
\hline
\end{tabular}

\begin{tabular}{|c|c|c|c|}
\hline $\mathbf{T}$ & Degree of freedom & P value & Implication \\
\hline 2.115 & 58 & 0.039 & Significant \\
\hline \multicolumn{4}{|c|}{ Table 5: Left parietooccipital sulcus } \\
\hline
\end{tabular}




\begin{tabular}{|c|c|c|c|}
\hline T & Degree of freedom & P value & Implication \\
\hline 1.677 & 57 & 0.099 & Not significant \\
\hline \multicolumn{3}{|c|}{ Table 6: Right parietooccipital sulcus } \\
\hline
\end{tabular}

\begin{tabular}{|c|c|c|c|}
\hline T & Degree of freedom & P value & Implication \\
\hline 0.314 & 58 & 0.754 & Not significant \\
\hline
\end{tabular}

Table 7: Third ventricle depth

\begin{tabular}{|c|c|c|c|}
\hline $\mathbf{T}$ & Degree of freedom & P value & Implication \\
\hline 0.1730 & 58 & 0.089 & Not significant \\
\hline
\end{tabular}

\begin{tabular}{|c|c|c|c|}
\hline $\mathbf{T}$ & Degree of freedom & P value & Implication \\
\hline 5.805 & 58 & 0.000 & Significant \\
\hline \multicolumn{4}{|c|}{ Table 9: Third ventricle width } \\
\hline
\end{tabular}

\section{AUTHORS:}

1. Vinu C. George

2. Jose Manuel

3. Biju Mohan

4. Indi Kusian

5. Sourabh A. P.

6. Arun V. J.

\section{PARTICULARS OF CONTRIBUTORS:}

1. Professor \& HOD, Department of Radiology, Azeezia Medical College Hospital, Meeyanoor.

2. Associate Professor, Department of Radiology, Azeezia Medical College Hospital, Meeyanoor.

3. Assistant Professor, Department of Radiology, Azeezia Medical College Hospital, Meeyanoor.

4. Assistant Professor, Department of Radiology, Azeezia Medical College Hospital, Meeyanoor.
5. House Surgeon, Department of Radiology, Azeezia Medical College Hospital, Meeyanoor.

6. Medical Student, Department of Radiology, Azeezia Medical College Hospital, Meeyanoor.

\section{NAME ADDRESS EMAIL ID OF THE} CORRESPONDING AUTHOR:

Dr. Vinu C. George,

Professor \& HOD,

Department of Radiology,

Azeezia Medical College Hospital,

Meeyanoor.

Email: anuraj04@gmail.com

Date of Submission: 05/09/2014.

Date of Peer Review: 06/09/2014.

Date of Acceptance: 09/09/2014.

Date of Publishing: 12/09/2014. 